\title{
Recombination activity of iron-gallium and iron-indium pairs in silicon
}

\author{
Jan Schmidta) \\ Institut für Solarenergieforschung Hameln/Emmerthal (ISFH), Am Ohrberg 1, D-31860 Emmerthal, \\ Germany \\ Daniel Macdonald \\ Department of Engineering, The Australian National University, Canberra ACT 0200, Australia
}

(Received 26 January 2005; accepted 13 April 2005; published online 6 June 2005)

\begin{abstract}
The recombination activity of iron-gallium (FeGa) and iron-indium (FeIn) pairs in crystalline silicon is studied by means of injection-dependent carrier lifetime measurements on Fe-implanted, Ga- and In-doped $p$-type silicon wafers of different resistivities $(0.3-15 \Omega \mathrm{cm})$. Compared to FeB pairs, FeGa and FeIn pairs are found to be much more effective recombination centers in $p$-type silicon. Using Shockley-Read-Hall statistics we determine the energy level $E_{t}$ of the FeGa-related center to be $0.20 \mathrm{eV}$ above the valence-band edge $E_{\nu}$. The strong recombination activity of FeGa is assigned to its large electron-capture cross section $\sigma_{n}$ of $4 \times 10^{-14} \mathrm{~cm}^{2}$. The hole-capture cross section $\sigma_{p}$ is $2 \times 10^{-14} \mathrm{~cm}^{2}$. For the FeIn-related recombination center, our measurements show that $E_{t}=E_{\nu}$ $+0.15 \mathrm{eV}, \sigma_{n}=3.5 \times 10^{-13} \mathrm{~cm}^{2}$, and $\sigma_{p}=1.5 \times 10^{-14} \mathrm{~cm}^{2}$. Strong illumination with white light is found to dissociate both types of pairs. Storage of the samples in the dark leads to a full repairing of FeGa and FeIn pairs. Lifetime changes measured before and after illumination can be used to determine the interstitial iron concentration in Ga- and In-doped silicon using calibration factors determined from the measured defect parameters. (C) 2005 American Institute of Physics.
\end{abstract}

[DOI: 10.1063/1.1929096]

\section{INTRODUCTION}

Boron-doped $p$-type silicon is the standard material in microelectronics and photovoltaics. However, a fundamental problem observed in solar cells made on this type of material is the light-induced performance degradation associated with the formation of boron-related defects. In particular in oxygen-rich Czochralski-grown $(\mathrm{Cz})$ silicon, a pronounced degradation in the solar cell efficiency can be observed, ${ }^{1}$ which could recently be attributed to the formation of a highly recombination-active boron-oxygen complex. ${ }^{2-5} \mathrm{Sev}$ eral strategies have been developed in recent years to reduce this degradation ${ }^{6}$ and one of the most successful methods to completely avoid the effect turned out to be the replacement of boron by alternative dopants, such as gallium and indium. ${ }^{2,5,7,8}$ The fact that the segregation coefficients of $\mathrm{Ga}$ and $\mathrm{In}$ in $\mathrm{Si}$ are well below that of $\mathrm{B}$ in $\mathrm{Si}$ causes a much stronger resistivity variation over the crystal height compared to B-doped Si crystals. However, in the case of Gadoped $\mathrm{Cz}-\mathrm{Si}$, the variation in resistivity has been demonstrated to be tolerable for the fabrication of high-efficiency solar cells. ${ }^{9}$ In fact, recent results of different groups have indicated that Ga-doped $\mathrm{Cz}-\mathrm{Si}$ is excellently suited as a base material for the production of high-efficiency solar cells. ${ }^{6}$ The segregation coefficient of In in Si is probably too low to allow for an application to solar cells. However, this has not been studied in detail so far. As neither gallium nor indium have been used in the microelectronics or photovoltaics industry as dopants in silicon up to now, there are only very few studies on the interaction of these shallow acceptors with other impurities, in particular iron, which is one of the most

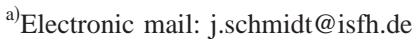

important contaminants in silicon. ${ }^{10,11}$ Furthermore, there is a severe lack of information concerning the recombination strength of FeGa and FeIn in Si. Up to now, there have only been two studies on the recombination activity of FeGa pairs in $\mathrm{Si},{ }^{12,13}$ both indicating a very strong recombination activity of FeGa pairs. No study has been published so far on the recombination activity of FeIn pairs in Si.

This paper aims at gaining a more detailed knowledge of the recombination parameters of $\mathrm{FeGa}$ and FeIn pairs in silicon to judge the susceptibility of Ga- and In-doped silicon to iron contamination. We study deliberately Fe-contaminated, Ga- and In-doped silicon wafers of different resistivities by means of injection-dependent recombination lifetime measurements. The measured data is fitted using ShockleyRead-Hall (SRH) theory, from which energy levels and capture cross sections are determined. In addition, the effect of illumination with white light on the iron-acceptor pairs is studied, showing that FeGa and FeIn pairs can be optically dissociated, similar to FeB pairs, ${ }^{14}$ which is of particular importance for solar cell applications. Finally, we calculate the calibration factors for the determination of the interstitial iron concentration in Ga- and In-doped silicon by measuring the lifetimes before and after pair dissociation. This highly sensitive iron-detection method has been developed and applied previously to boron-doped silicon only. ${ }^{15,16}$

\section{IRON-ACCEPTOR PAIRS IN SILICON}

In crystalline $p$-type silicon, highly mobile iron atoms form electrically active pairs with shallow acceptors such as boron, aluminum, gallium, and indium. ${ }^{17}$ The configurational structure of these pairs has mainly been studied by electron paramagnetic resonance (EPR), and the energy level and 
capture cross sections of electrons and holes by deep-level transient spectroscopy (DLTS). A comprehensive review of the properties of iron and iron-acceptor pairs in silicon has recently been published by Istratov et al. ${ }^{11}$

The driving force for the formation of iron-acceptor pairs is the electrostatic attraction between the positively charged interstitial iron $\left(\mathrm{Fe}_{i}^{+}\right)$and the negatively charged substitutional acceptor $\left(A_{s}^{-} ; A=\mathrm{B}, \mathrm{Al}, \mathrm{Ga}, \mathrm{In}\right)$. As interstitial iron is only in a positive-charge state if the Fermi level is below the energy level of $\mathrm{Fe}_{i}^{0 /+}$ at $E_{\nu}+0.38 \mathrm{eV}$, the pair formation cannot be observed in $n$-Si partly compensated with boron. ${ }^{18}$ First-principles calculations suggest that, apart from the ionic component, covalency involving the $\mathrm{Fe}_{i}, A_{s}$, and the surrounding $\mathrm{Si}$ atoms probably also plays an important role in the bonding of the pairs. ${ }^{19}$ Theoretical results on reaction kinetics, deep levels, and pair structures suggest that the size of the substitutional acceptor atoms is fundamental for the $\mathrm{Fe}_{i} A_{s}$ pair energetics. ${ }^{20}$

As known from EPR studies, ${ }^{11} \mathrm{Fe}_{i} A_{s}$ pairs have two structural configurations with trigonal and orthorhombic symmetries, each of them existing in negative-, neutral-, and positive-charge states, resulting in four deep levels for each $\mathrm{Fe}_{i} A_{s}$ pair (two acceptor and two donor states). $\mathrm{Fe}_{i}$ is placed either at the nearest-neighbor position to $A_{s}$ along the $\langle 111\rangle$ direction in the pairs with trigonal symmetry or the nextnearest-neighbor tetrahedral interstitial sites along the $\langle 100\rangle$ direction in the pairs with orthorhombic symmetry. It has been shown by means of experiments and theory that $\mathrm{FeB}$, $\mathrm{FeAl}$, and $\mathrm{FeGa}$ pairs are stable in the trigonal configuration and metastable in the orthorhombic configuration, whereas FeIn is stable in the orthorhombic configuration and unstable in the trigonal configuration. ${ }^{21}$ Kimerling et $a .^{21}$ explained the different behavior of FeIn pairs compared to the other iron-acceptor pairs with the competition between repulsive elastic and attractive electrostatic energy terms (note that In is the acceptor with the largest covalent radius).

One shallow and one deeper donor level have been measured for each $\mathrm{Fe}_{i} A_{s}$ pair, with the deeper level being associated with the $\langle 111\rangle$ trigonal pair configuration and the shallower level relates to the $\langle 100\rangle$ orthorhombic configuration. ${ }^{11}$ It was also found that the levels become deeper with increasing ionization energy of the acceptors and the covalent radius of the acceptors. ${ }^{22}$ These experimental results are in agreement with theoretical calculations by Zhao et al. ${ }^{23}$ who accounted for the lattice relaxation due to the different sizes of the acceptor atoms. The reported energy levels for the different $\mathrm{Fe}_{i} A_{s}$ pairs measured by capacitance spectroscopy are listed in Table I. As can be seen from Table I, acceptor levels have only been found for $\mathrm{FeB}$ pairs. For $\mathrm{FeAl}, \mathrm{FeGa}$, and FeIn no acceptor levels have been measured so far by capacitance spectroscopy techniques.

There is only limited information available on which of the existing energy levels of $\mathrm{Fe}_{i} A_{s}$ pairs are the dominant recombination centers. Only for the FeB pair have reliable carrier lifetime measurements shown that the acceptor level at $E_{c}-(0.26 \pm 0.03) \mathrm{eV}$, related to the stable trigonal-pair configuration, is the dominant recombination center. ${ }^{24-26}$ For $\mathrm{FeAl}$ and the $\mathrm{FeGa}$ it can only be conjectured that the deep donor levels at $E_{\nu}+(0.20 \pm 0.01)$ and $E_{\nu}$
TABLE I. Electrical levels of iron-acceptor pairs in silicon determined using capacitance spectroscopy. The data are taken from Refs. 11-13. Note that the uncertainty in the energy levels reflects only the scatter in the experimental data reported by different groups and not possible additional measurement errors.

\begin{tabular}{|c|c|c|c|}
\hline Defect, symmetry & $\begin{array}{l}\text { Donor }(\mathrm{d}) / \\
\text { acceptor }(\mathrm{a})\end{array}$ & $\begin{array}{c}\text { Energy level } \\
E_{t} \\
(\mathrm{eV})\end{array}$ & $\begin{array}{l}\text { Stable }(\mathrm{s}) / \\
\text { metastable }(\mathrm{ms})\end{array}$ \\
\hline$(\mathrm{FeB})^{0 /+}$, trigonal & d & $E_{\nu}+0.1$ & $\mathrm{~s}$ \\
\hline$(\mathrm{FeB})^{-/ 0}$, trigonal & a & $E_{c}-(0.26 \pm 0.03)$ & $\mathrm{s}$ \\
\hline $\begin{array}{l}(\mathrm{FeB})^{0 /+} \text {, orthorhombic } \\
\text { (tentatively) }\end{array}$ & d & $E_{\nu}+0.07$ & $\mathrm{~ms}$ \\
\hline $\begin{array}{l}(\mathrm{FeB})^{-/ 0} \text {, orthorhombic } \\
\text { (tentatively) }\end{array}$ & $\mathrm{a}$ & $E_{c}-0.43$ & $\mathrm{~ms}$ \\
\hline$(\mathrm{FeAl})^{0 /+}$, trigonal & $d$ & $E_{\nu}+(0.20 \pm 0.01)$ & s \\
\hline$(\mathrm{FeAl})^{0 /+}$, orthorhombic & $\mathrm{d}$ & $E_{\nu}+0.13$ & $\mathrm{~ms}$ \\
\hline$(\mathrm{FeGa})^{0 /+}$, trigonal & d & $E_{\nu}+(0.225 \pm 0.015)$ & $\mathrm{s}$ \\
\hline$(\mathrm{FeGa})^{0 /+}$, orthorhombic & d & $E_{\nu}+(0.125 \pm 0.025)$ & $\mathrm{ms}$ \\
\hline$(\mathrm{FeIn})^{0 /+}$, trigonal & d & $E_{\nu}+0.27$ & $\mathrm{~ms}$ \\
\hline$(\mathrm{FeIn})^{0 /+}$, orthorhombic & d & $E_{\nu}+0.15$ & $\mathrm{~s}$ \\
\hline
\end{tabular}

$+(0.225 \pm 0.015) \mathrm{eV},{ }^{11-13}$ respectively, both related to the stable trigonal configuration of these pairs, are the dominant recombination centers. For FeIn, on the other hand, the shallow donor level at $E_{\nu}+0.15 \mathrm{eV}$ is probably the dominant recombination center, ${ }^{27}$ as it is associated with the stable orthorhombic configuration of the pair.

\section{EXPERIMENTAL DETAILS}

\section{A. Sample preparation}

Gallium- and indium-doped (100)-oriented Czochralskigrown 6-in. $p$-type silicon wafers of 300- $\mu \mathrm{m}$ thickness were chosen in this study. Ga-doped Si wafers of three different resistivities $(0.3,3$, and $10 \Omega \mathrm{cm})$ and $I n$-doped $\mathrm{Si}$ wafers of $14.5-\Omega \mathrm{cm}$ resistivity were used. The interstitial oxygen content of all $\mathrm{Cz}-\mathrm{Si}$ wafers used in this study, as determined by infrared absorption, is in the range of $7 \times 10^{17}-8$ $\times 10^{17} \mathrm{~cm}^{-3}$, whereas the substitutional carbon content is below the detection limit of $5 \times 10^{15} \mathrm{~cm}^{-3}$. Reference samples were fabricated on $1-\Omega \mathrm{cm}$ boron-doped (100)-oriented float-zone (FZ) silicon wafers.

Prior to ion implantation all wafers were cut in 4.5 $\times 4.5-\mathrm{cm}^{2}$ sized samples and subsequently RCA cleaned. The implantation area was defined by means of a silicon aperture allowing an implantation area of $3.2 \times 3.3 \mathrm{~cm}^{2}$. The most abundant iron isotope ${ }^{56} \mathrm{Fe}$ was selected for implantation, with a dose of $10^{11} \mathrm{~cm}^{-2}$, corresponding to an implantation time of $\sim 10 \mathrm{~s}$. A low implantation energy of $70 \mathrm{keV}$ was chosen to minimize lattice damage, which can act as unwanted gettering sites during the later annealing treatment.

After implantation the samples were again given an RCA clean to remove possible metal contaminants from the surfaces. Annealing was then performed at $900{ }^{\circ} \mathrm{C}$ for $1 \mathrm{~h}$ in nitrogen atmosphere in a TCA-cleaned quartz-tube furnace to distribute the iron uniformly throughout the wafers. For a wafer thickness of $300 \mu \mathrm{m}$ and an implantation dose of 
TABLE II. Energy levels and capture cross sections for iron-related recombination centers in crystalline silicon. The parameters for $\mathrm{Fe}_{i}$ and $\mathrm{FeB}$ are taken from the literature (Refs. 11 and 26), whereas the defect parameters for FeGa and FeIn have been determined in this study. Also shown are approximations for the SRH lifetimes under low- and high-injection conditions.

\begin{tabular}{|c|c|c|c|c|c|c|}
\hline $\begin{array}{c}\text { Recombination } \\
\text { center }\end{array}$ & $\begin{array}{c}\text { Energy level } \\
E_{t}(\mathrm{eV})\end{array}$ & $\begin{array}{c}\text { Electron- } \\
\text { capture cross } \\
\text { section } \\
\sigma_{n}\left(\mathrm{~cm}^{2}\right)\end{array}$ & $\begin{array}{c}\text { Hole- } \\
\text { capture cross } \\
\text { section } \\
\sigma_{p}\left(\mathrm{~cm}^{2}\right)\end{array}$ & $\sigma_{n} / \sigma_{p}$ & $\begin{array}{c}\text { Low-injection } \\
\tau_{\mathrm{SRH}}\end{array}$ & $\begin{array}{c}\text { High-injection } \\
\tau_{\mathrm{SRH}}\end{array}$ \\
\hline $\mathrm{Fe}_{i}$ & $E_{\nu}+0.38$ & $4 \times 10^{-14}$ & $7 \times 10^{-17}$ & 571 & $\tau_{n 0}$ & $\tau_{p 0}$ \\
\hline FeB acceptor & $E_{c}-0.26$ & $1.4 \times 10^{-14}$ & $1.1 \times 10^{-15}$ & 13 & $\tau_{p 0}\left(n_{1} / N_{A}\right)+\tau_{n 0}$ & $\tau_{p 0}$ \\
\hline FeGa donor & $E_{\nu}+0.20$ & $4 \times 10^{-14}$ & $2 \times 10^{-14}$ & 2 & $\tau_{n 0}\left(1+p_{1} / N_{A}\right)$ & $\tau_{n 0}+\tau_{p 0}$ \\
\hline FeIn donor ${ }^{a}$ & $E_{\nu}+0.15$ & $3.5 \times 10^{-13}$ & $1.5 \times 10^{-14}$ & 23 & $\tau_{n 0}\left(1+p_{1} / N_{A}\right)$ & $\tau_{p 0}$ \\
\hline
\end{tabular}

${ }^{\mathrm{a}}$ Tentative results.

$10^{11} \mathrm{~cm}^{-2}$, we calculate an interstitial iron concentration of $3.3 \times 10^{12} \mathrm{~cm}^{-3}$, which is well below the solubility limit of $\sim 5 \times 10^{13} \mathrm{~cm}^{-3}$ of interstitial iron in silicon. ${ }^{11}$ Hence, we may assume that in our samples no unwanted formation of iron precipitates occurs. The interstitial iron concentration determined from the light-stimulated change in lifetime measured on the boron-doped reference samples using the calibration factor recently published by Macdonald et al. ${ }^{16}$ is about $5 \%$ higher than the $\mathrm{Fe}_{i}$ concentration calculated from the implantation dose. This deviation is well within the uncertainty range of the experiment.

In order to remove any residual surface damage caused by the ion implantation, approximately $5 \mu \mathrm{m}$ of silicon were etched off both wafer surfaces in an acid-etching solution. After another RCA clean both wafer surfaces were passivated by means of plasma-enhanced chemical-vapordeposited silicon nitride films. This passivation method has been shown to provide very low surface recombination velocities $<10 \mathrm{~cm} / \mathrm{s},{ }^{28}$ which was verified on nonimplanted control wafers of the same type. Hence, surface recombination can be completely neglected for all iron-implanted samples investigated in this study. Also, the relatively low deposition temperature of $400{ }^{\circ} \mathrm{C}$ for a few minutes avoids potential precipitation problems that might occur if higher temperature processes, such as oxidation, have been applied.

\section{B. Injection-dependent lifetime measurements}

The contactless quasi-steady-state photoconductance (QSSPC) method developed by Sinton and Cuevas ${ }^{29}$ was used to measure the injection-dependent lifetime curves at room temperature $(T=300 \mathrm{~K})$. In this method, a coil in a resonant circuit, operating at $13.56 \mathrm{MHz}$, couples to the conductivity of the sample. The output voltage of the bridge circuit is linear in wafer conductance over the entire measurement range. Reference wafers of known dark conductance are used to calibrate the rf bridge circuit. A conventional photo-flash light source, having a slow decay time of about $2 \mathrm{~ms}$, generates electron-hole pairs in the silicon sample. As the light pulse varies very slowly compared to the effective carrier lifetime of the sample, the latter is approximately under steady-state conditions during the complete 8 -ms illumination time. The time-dependent light intensity, measured with a calibrated solar cell, as well as the rf-bridge output voltage are recorded on a digital oscilloscope and transferred to a computer for analysis. From the measured photoconductance and light-intensity data, the effective lifetime of the sample can then be calculated as a function of the injection density. ${ }^{30}$ One of the main advantages of the QSSPC technique over transient decay approaches is that it allows very low lifetimes to be measured over a broad injection range without fast electronics or short light pulses. ${ }^{29}$ As the detection area equals approximately the coil area of $\sim 3 \mathrm{~cm}^{2}$, the implanted area of the measured silicon samples completely covers the detection area.

\section{SHOCKLEY-READ-HALL LIFETIME}

Provided the defect density $N_{t}$ is sufficiently small, ${ }^{31}$ the recombination lifetime can be modeled by traditional $\mathrm{SRH}$ statistics. ${ }^{32,33}$ For interstitial iron in silicon this prerequisite is virtually always valid because of its relatively low solubility in silicon. Assuming negligible trapping and, hence, equal excess electron and hole concentrations $(\Delta n=\Delta p)$, the SRH lifetime associated with a single-level defect in $p$-type silicon with an acceptor concentration $N_{A}$ is given by ${ }^{34}$

$$
\tau_{\mathrm{SRH}}=\frac{\tau_{n 0}\left(N_{A}+p_{1}+\Delta n\right)+\tau_{p 0}\left(n_{1}+\Delta n\right)}{N_{A}+\Delta n},
$$

where the electron- and hole-capture time constants $\tau_{n 0}$ and $\tau_{p 0}$ are related to the defect density $N_{t}$, the thermal velocity $v_{\text {th }}=1.1 \times 10^{7} \mathrm{~cm} \mathrm{~s}^{-1}$ (at $\left.T=300 \mathrm{~K}\right),{ }^{35}$ and the electron- and hole-capture cross sections $\sigma_{n}$ and $\sigma_{p}$ via $\tau_{n 0}=\left(v_{\mathrm{th}} N_{t} \sigma_{n}\right)^{-1}$ and $\tau_{p 0}=\left(v_{\mathrm{th}} N_{t} \sigma_{p}\right)^{-1}$. The densities $n_{1}$ and $p_{1}$ equal the equilibrium electron and hole densities when the defect energy level $E_{t}$ coincides with the Fermi level:

$$
n_{1}=N_{c} \exp \left(-\frac{E_{c}-E_{t}}{k T}\right), \quad p_{1}=N_{v} \exp \left(-\frac{E_{t}-E_{v}}{k T}\right) .
$$

Values for the effective densities of states in the conduction and valence band at $300 \mathrm{~K}$ are taken as $N_{c}=2.86$ $\times 10^{19} \mathrm{~cm}^{-3}$ and $N_{\nu}=3.10 \times 10^{19} \mathrm{~cm}^{-3} \cdot{ }^{36}$

As can directly be seen from Eqs. (1) and (2), under low-injection conditions (i.e., $\Delta n \ll N_{A}$ ), the SRH lifetime approaches a constant value $\tau_{\mathrm{SRH}}=\tau_{n 0}$ for a deep-level center for which $n_{1}$ and $p_{1}$ in Eq. (1) can be neglected. This is the case for interstitial iron. For a shallow-level center, such as iron-acceptor pairs, for which either $n_{1}$ or $p_{1}$ cannot be neglected, the low-injection SRH lifetime depends on the doping concentration, as shown in Table II. Under high-injection 


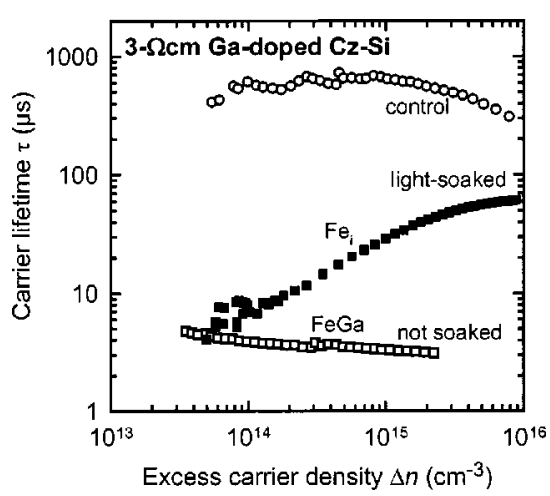

FIG. 1. Injection-dependent lifetime measurements for an iron-implanted $3-\Omega \mathrm{cm}$ Ga-doped $p$-type Si sample before (open squares) and after (closed squares) light soaking for $1 \mathrm{~min}$ at an intensity of $0.7 \mathrm{~W} / \mathrm{cm}^{2}$ using halogen lamps. Also shown is the measured lifetime of a nonimplanted control sample (open circles).

conditions (i.e., $\Delta n \gg N_{A}, n_{1}, p_{1}$ ), the SRH lifetime is the same for deep- and shallow-level centers $\tau_{\mathrm{SRH}}=\tau_{n 0}+\tau_{p 0}$.

Note that in this study we only account for SRH recombination and neglect all other recombination processes, such as Auger and radiative recombinations. It has been shown before $^{25}$ that Auger and radiative recombinations can indeed be neglected in iron-contaminated silicon samples as investigated in this study if the injection range is restricted to lowand medium-injection levels.

\section{RESULTS AND DISCUSSION}

\section{A. FeGa pairs}

The two lower curves (open and closed squares) in Fig. 1 show injection-dependent lifetime measurements for an $\mathrm{Fe}$ contaminated $3-\Omega \mathrm{cm}$ Ga-doped $p$-Si wafer (Fe concentration $3.3 \times 10^{12} \mathrm{~cm}^{-3}$ ) before and after a 1-min illumination by halogen lamps at a light intensity of $\sim 0.7 \mathrm{~W} / \mathrm{cm}^{2}$. The upper curve (circles) corresponds to an unimplanted control wafer of the same type that has been subjected to the same temperature and cleaning treatments as the Fe-contaminated samples. The relatively high lifetime of the control wafer around $700 \mu \mathrm{s}$ indicates that very little, if any, additional contamination has been introduced into our samples during the $900{ }^{\circ} \mathrm{C}$ anneal. The relatively low $\mathrm{Fe}$ concentration of $3.3 \times 10^{12} \mathrm{~cm}^{-3}$ reduces the carrier lifetime drastically by two orders of magnitude to values around $4 \mu \mathrm{s}$ (open squares). Since these lifetime measurements have been performed after storing the wafers for more than one week in the dark, it can be assumed that all $\mathrm{Fe}$ atoms have formed pairs with $\mathrm{Ga}$ and, hence, the very low lifetimes measured in the Fe-contaminated $\mathrm{Ga}$-doped $\mathrm{Si}$ wafers can be attributed to FeGa pairs. As mentioned in Sec. II, generally, iron-acceptor pairs are considered as equilibrium states in $p$-type silicon with Fermi level below the energy level of interstitial $\mathrm{Fe}$ (i.e., for $E_{F}<E_{\nu}+0.38 \mathrm{eV}$ ). This is so because of the strong electrostatic attraction of the positively charged iron atoms and the negatively charged acceptor atoms. Compared to B-doped $p$-Si wafers with comparable doping concentration and $\mathrm{Fe}$ contamination, the lifetimes in Ga-doped $p$-Si are much lower. For example, studying $5-\Omega \mathrm{cm}$ B-doped $p$-Si wafers with $\mathrm{Fe}$ concentrations around $3.5 \times 10^{12} \mathrm{~cm}^{-3}$,
Macdonald et al. ${ }^{25}$ measured lifetimes around $20 \mu$ s, which is a factor of five higher compared to the lifetimes measured on comparable Ga-doped wafers in this work. This comparison clearly indicates that $\mathrm{FeGa}$ pairs are much more effective recombination centers than $\mathrm{FeB}$ pairs.

Illumination increases the lifetimes of all $\mathrm{Fe}$ contaminated Ga-doped $p$-Si samples examined in this study. As can be seen from Fig. 1, the lifetime depends only very weakly on the excess carrier concentration before illumination, whereas after light soaking the lifetime shows a pronounced increase with increasing injection density. It will be shown below (see Fig. 4) that all our injection-dependent lifetime curves measured after illumination can be modeled very accurately using Eq. (1), in combination with the defect parameters for $\mathrm{Fe}_{i}$ reported in the literature (see Table II). Hence, the observed lifetime changes can be interpreted as a light-stimulated dissociation of $\mathrm{FeGa}$ pairs into $\mathrm{Fe}_{i}$ and $\mathrm{Ga}_{s}$. We assume that the pair-splitting mechanism is the same as the one known from the light-stimulated FeB dissociation, ${ }^{14,37}$ that is, a recombination-enhanced dissociation. As a consequence, only minority carriers are necessary to trigger the pair dissociation, while the photons themselves are not directly involved in the reaction. The observation of a lightstimulated dissociation of $\mathrm{FeGa}$ pairs is particularly relevant for solar cells made on Ga-doped silicon wafers. As solar cells operate under strong illumination, FeGa pairs will usually dissociate, leaving only isolated $\mathrm{Fe}_{i}$ atoms to influence the recombination lifetime in the cell base.

It can be seen from Fig. 1 that the two lifetime curves measured before and after light soaking approach each other with decreasing injection density. Our measurements suggest that there is a crossover point of both curves well below an excess carrier density of $10^{14} \mathrm{~cm}^{-3}$. Unfortunately, we were not able to observe this crossover point directly as all our measurements at low-injection levels were hampered by minority-carrier trapping effects, resulting in a drastic increase of the photoconductance signal at low-injection levels, which is not related to an increasing recombination lifetime. ${ }^{38}$ Such trapping effects can be particularly pronounced in $\mathrm{Cz}-\mathrm{Si}$ wafers due to the high oxygen content, which was analyzed in detail recently. ${ }^{39}$ Hence, in this study we have deleted all low-injection lifetime data corrupted by trapping effects.

In order to determine the energy level and the electronand hole-capture cross sections of the FeGa recombination center, we have studied Ga-doped $p$-Si wafers of different doping concentrations. Figure 2 shows the resulting injection-dependent lifetime curves measured on $\mathrm{Fe}$ contaminated Ga-doped $p$-Si wafers of three different resistivities $(0.3,3$, and $10 \Omega \mathrm{cm})$. As the wafers were stored in the dark for more than one week, we assume that all $\mathrm{Fe}$ atoms are paired with $\mathrm{Ga}$ atoms and, hence, $[\mathrm{FeGa}]=3.3$ $\times 10^{12} \mathrm{~cm}^{-3}$.

The pronounced doping-concentration dependence of the low-injection lifetime in Fig. 2 indicates that the FeGa recombination center has a relatively shallow energy level in the Si band gap. As discussed in Sec. II, there is strong evidence from the literature for the existence of two electrical levels of FeGa pairs in silicon. ${ }^{11-13}$ One state occurs be- 


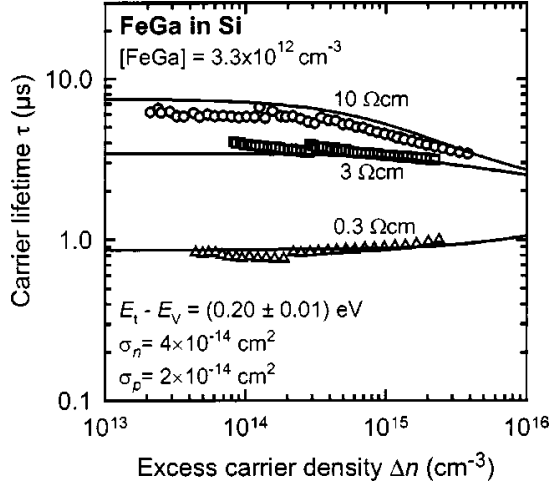

FIG. 2. Injection-dependent lifetime measurements (symbols) and SRH fits (solid lines) for iron-contaminated Ga-doped Si samples of three different resistivities $(0.3,3$, and $10 \Omega \mathrm{cm})$. The total iron concentration of all samples is $3.3 \times 10^{12} \mathrm{~cm}^{-3}$. As the samples were stored in the dark for more than one week prior to the measurements, all interstitial iron atoms are paired with gallium.

tween $E_{\nu}+0.21$ and $E_{\nu}+0.24 \mathrm{eV}$ and the other one between $E_{\nu}+0.10$ and $E_{\nu}+0.15 \mathrm{eV} .^{11-13}$ The two states are donor levels associated with the stable trigonal and the metastable orthorhombic configurations of the FeGa pair, respectively. In Sec. IV it was shown that the recombination via shallowlevel centers with the energy level close to the valence-band edge results in a low-injection SRH lifetime $\tau_{\mathrm{SRH}, \mathrm{li}}$, depending on the doping concentration $N_{A}$ as $\tau_{\mathrm{SRH}, \mathrm{li}}=\tau_{n 0}$ $+\tau_{n 0} p_{1} / N_{A}$. Hence, a linear fit of the measured low-injection lifetimes $\tau_{\mathrm{SRH}, \mathrm{li}}$ taken from Fig. 2 as a function of the inverse doping concentration $1 / N_{A}$ gives $\tau_{n 0}$ and $p_{1}$ (see Fig. 3). From the intersection of the linear fit function in Fig. 3 with the $y$ axis, we obtain for the electron-capture time constant $\tau_{n 0}=(0.7 \pm 0.1) \mu \mathrm{s}$. Using $\sigma_{n}=\left(v_{\text {th }} \tau_{n 0} N_{t}\right)^{-1}$ with the defect density $N_{t}=3.3 \times 10^{12} \mathrm{~cm}^{-3}$, an electron-capture cross section of $\sigma_{n}=(3.9 \pm 0.6) \times 10^{-14} \mathrm{~cm}^{2}$ is obtained. The slope of the linear fit in Fig. 3 equals the product of $\tau_{n 0}$ and $p_{1}$. As $\tau_{n 0}$ is known, the slope directly gives $p_{1}=1 \times 10^{16}-2$ $\times 10^{16} \mathrm{~cm}^{-3}$. In the determination of the slope, the error is relatively large as the low-injection lifetimes of the Gadoped Si wafers in Fig. 3 are not on a perfect straight line. However, as according to Eq. (2) the energy level $E_{t}-E_{\nu}$ of the recombination center is given by $k T \ln \left(p_{1} / N_{\nu}\right)$, the relatively large error in $p_{1}$ results only in a small energy level

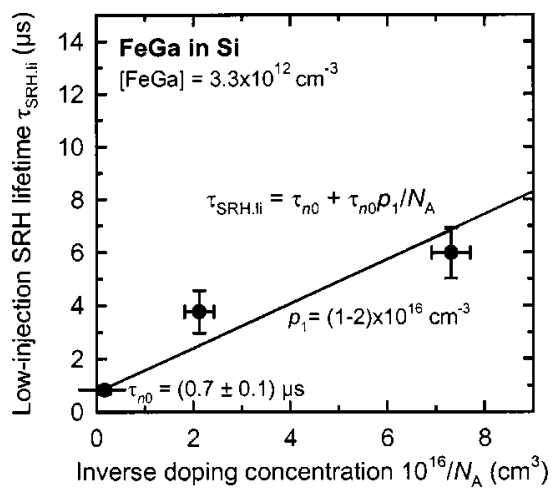

FIG. 3. Measured low-injection lifetimes $\tau_{\mathrm{SRH}, \mathrm{li}}$ (circles) obtained from Fig. 2 as a function of the inverse doping concentration $1 / N_{A}$ and linear fit (line) for FeGa pairs in $p$-type Si.

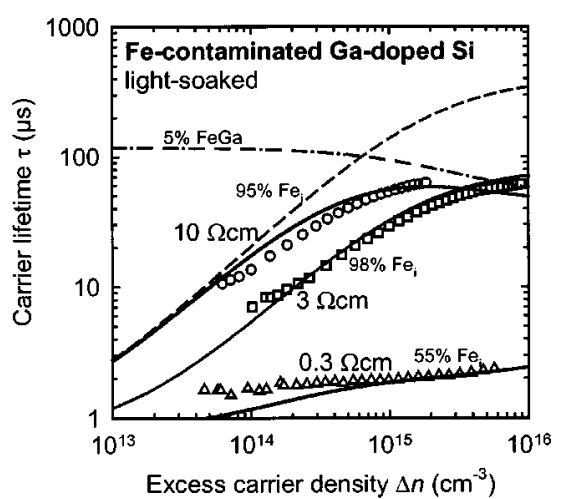

FIG. 4. Injection-dependent lifetime measurements (symbols) and SRH fits (solid lines) for iron-contaminated Ga-doped Si samples of three different resistivities $(0.3,3$, and $10 \Omega \mathrm{cm})$ after light soaking. The total iron concentration of $3.3 \times 10^{12} \mathrm{~cm}^{-3}$ is the same for all three samples. Different concentrations of $\mathrm{Fe}_{i}$ and $\mathrm{FeGa}$ pairs were used to generate the fits (given in percent of the total iron concentration).

uncertainty of $E_{t}=E_{\nu}+(0.20 \pm 0.01) \mathrm{eV}$. This energy level is in good agreement with the DLTS results of Ciszek and Wang, ${ }^{12}$ who determined $E_{t}=E_{\nu}+0.21 \mathrm{eV}$ for the deep donor level of the FeGa pair. Hence, our results strongly suggest that the deep donor level dominates the recombination through $\mathrm{FeGa}$ pairs at room temperature. As discussed in Sec. II, the deep donor level corresponds to the trigonal configuration, which is the stable form of FeGa pairs. ${ }^{11}$ This is important to note, as in the determination of $\sigma_{n}$ we have assumed that all $\mathrm{FeGa}$ pairs are in the same state. The relatively large $\sigma_{n}$ value of $\sim 4 \times 10^{-14} \mathrm{~cm}^{2}$ is the reason for the strong recombination activity of FeGa pairs in silicon. Interestingly, the electron-capture cross sections for $\mathrm{FeGa}$ and for isolated $\mathrm{Fe}_{i}$ are practically identical (see Table II).

The hole-capture cross section $\sigma_{p}$ for the FeGa center is determined by simultaneously fitting the SRH Eq. (1) to the injection-dependent lifetime curves in Fig. 2. As the energy level $E_{t}$ and the electron-capture cross section $\sigma_{n}$ are known from the previous analysis of the low-injection lifetime data and the recombination center density $N_{t}$ is a fixed parameter, $\sigma_{p}$ is the only free fit parameter in Eq. (1). From the fit of the injection-dependent lifetime curves in Fig. 2 (solid lines) using the $\sigma_{n}$ and $E_{t}$ values given in Table II, we determine $\sigma_{p}=(2 \pm 1) \times 10^{-14} \mathrm{~cm}^{2}$. As the high-injection SRH lifetime $\tau_{\mathrm{SRH}, \mathrm{hi}}$ equals $\tau_{n 0}+\tau_{p 0}$, the lifetimes in Fig. 2 are only moderately sensitive to variations in $\sigma_{p}$ within the low- to medium-injection range of our lifetime measurements. Hence, the uncertainty range is larger for $\sigma_{p}$ than for $\sigma_{n}$. There are only a few studies in the literature reporting holecapture cross sections for the deep donor level of FeGa in silicon, and the scatter in the $\sigma_{p}$ data is around one order of magnitude. ${ }^{11}$ The $\sigma_{p}$ determined in this work is in excellent agreement with the DLTS results of Wünstel and Wagner ${ }^{40}$ and Beljakowa et al., ${ }^{13}$ who measured a $\sigma_{p}$ value of 2 $\times 10^{-14} \mathrm{~cm}^{2}$.

Figure 4 shows the injection-dependent lifetime measurements of the same samples as shown in Fig. 2 after illumination with white light for $1 \mathrm{~min}$ at $0.7 \mathrm{~W} / \mathrm{cm}^{2}$. The measured curves can be accurately modeled, assuming two recombination centers to be present. One center is interstitial 
iron with the defect parameters taken from the literature and the second center is the deep donor level of the iron-gallium pair analyzed in this work (all defect parameters used in the modeling are given in Table II). The SRH lifetime of each center is calculated using Eq. (1), and the inverse SRH lifetimes are added to obtain the inverse total lifetime. As the total iron concentration is known from the implantation dose and the wafer thickness (i.e., $\left[\mathrm{Fe}_{i}\right]+[\mathrm{FeGa}]=3.3$ $\times 10^{12} \mathrm{~cm}^{-3}$ ), the only free parameter in the fitted curves, shown as solid lines in Fig. 4, is the ratio of $\mathrm{Fe}_{i}$ to $\mathrm{FeGa}$ concentrations. Note that we have assumed again that all FeGa pairs are in the stable trigonal state only. The dashed line in Fig. 4 shows the $\mathrm{Fe}_{i}$-related contribution to the total lifetime and the dash-dotted line the SRH lifetime of the FeGa center, both for the $10-\Omega \mathrm{cm}$ sample. For the 10 - and the $3-\Omega \mathrm{cm}$ Ga-doped $\mathrm{Si}$ wafers, the fits clearly prove that practically all FeGa pairs, that is, more that $95 \%$, have been dissociated during the 1-min illumination. In the case of the low-resistivity $0.3-\Omega \mathrm{cm} \mathrm{Si}$ wafer, however, $55 \%$ of $\mathrm{Fe}_{i}$ and $45 \%$ of $\mathrm{FeGa}$ pairs are present. We attribute this lower $\mathrm{Fe}_{i}$ concentration to the very fast association of FeGa pairs in the highly doped material. Hence, during the time interval between the light soaking and the lifetime measurement $(\sim 1 \mathrm{~min})$, a large number of $\mathrm{FeGa}$ pairs may reform. As for the FeB pair, the association time constant $\tau_{\text {as }}$ can, assuming Coulombic attraction between the pairing species only (in our case $\mathrm{Fe}_{i}^{+}$and $\mathrm{Ga}_{s}^{-}$), be expressed as ${ }^{37}$

$$
\tau_{\mathrm{as}}=\frac{\varepsilon \varepsilon_{0} k T}{q^{2} N_{A} D\left(\mathrm{Fe}_{i}\right)},
$$

where $D\left(\mathrm{Fe}_{i}\right)$ is the iron diffusivity in silicon, $\varepsilon$ is the dielectric constant of silicon, and $\varepsilon_{0}$ is the vacuum permittivity. In order to estimate the order of magnitude of $\tau_{\text {as }}$ for the three samples in Fig. 4, we use Eq. (3) with a room-temperature iron diffusivity of $D\left(\mathrm{Fe}_{i}\right)=10^{-14} \mathrm{~cm}^{2} / \mathrm{s}^{41}$ The association time constants calculated for the $10-$ and the $3-\Omega \mathrm{cm} \mathrm{Si}$ wafers are 207 and $60 \mathrm{~min}$, respectively, while that of the 0.3$\Omega \mathrm{cm}$ sample is only $5 \mathrm{~min}$. This is in good agreement with the partial repairing observed for the $0.3-\Omega \mathrm{cm}$ wafer, taking place in the interval between the 1-min illumination and the lifetime measurement. Note that the scatter in the iron diffusivities reported in the literature is very large ${ }^{11}$ and, hence, the determined association time constants can only be regarded as rough estimates.

\section{B. Feln pairs}

Figure 5 shows the injection-dependent lifetime measurements for an Fe-contaminated $14.5-\Omega \mathrm{cm}$ In-doped $p-\mathrm{Si}$ wafer before and after light soaking. The circles show the lifetime of a control wafer of the same type, which, apart from the ion implantation, was given exactly the same treatment as the Fe-contaminated $\mathrm{Si}$ wafer. The high lifetime $(\sim 750 \mu \mathrm{s})$ of the control wafer indicates that no additional contamination was introduced during processing. The lifetime measurement before light soaking was performed after storing the wafer for more than one week in the dark. Hence, it can be assumed that all mobile interstitial $\mathrm{Fe}_{i}$ atoms have formed pairs with immobile substitutional $\operatorname{In}_{s}$ and the very

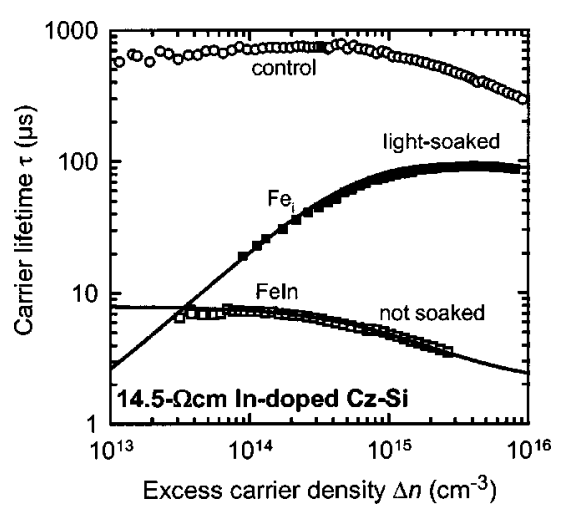

FIG. 5. Injection-dependent lifetime measurements for an iron-implanted $14.5-\Omega \mathrm{cm}$ In-doped Si sample before (open squares) and after (closed squares) light soaking and SRH fits (solid lines). Also shown is the measured lifetime of a nonimplanted control sample (open circles).

low lifetimes $(\sim 7 \mu \mathrm{s})$ measured in the "not soaked" state can be attributed to FeIn pairs. These measurements clearly indicate that FeIn is a very effective recombination center, as is FeGa. Unfortunately, we are not able to determine the exact energy level of the FeIn-related recombination center, because In-doped $p$-Si wafers of only one resistivity were available in this study. However, the measured injectiondependent lifetime curve in the not-soaked state in Fig. 5 can only be fitted by Eq. (1) if the energy level is closer than $\sim 0.26 \mathrm{eV}$ to one of the band edges. In the literature, two FeIn-related donor levels at $E_{\nu}+0.15$ and $E_{\nu}+0.27 \mathrm{eV}$ have been obtained using DLTS. ${ }^{27}$ However, in contrast to FeGa pairs, for FeIn the shallow donor level, assigned to the orthorhombic configuration, was identified as the stable state, whereas the metastable trigonal configuration corresponds to the deep donor state. In order to estimate the capture cross sections for the FeIn pair, we assume that all FeIn pairs are in the stable configuration and the energy level is $E_{t}=E_{\nu}$ $+0.15 \mathrm{eV}$, as reported by Chantre and Kimerling. ${ }^{27}$ Fitting Eq. (1) to the measured lifetime data in Fig. 5 (open squares) results in a very large $\sigma_{n}$ value of $3.5 \times 10^{-13} \mathrm{~cm}^{2}$ and a $\sigma_{p}$ of $1.5 \times 10^{-14} \mathrm{~cm}^{2}$. In spite of the fact that the energy level of $E_{\nu}+0.15 \mathrm{eV}$ is very shallow, due to the very large electroncapture cross section, FeIn is a highly effective recombination center. Note that our results exclude the energy levels at $E_{c}-0.32$ and $E_{c}-0.39 \mathrm{eV}$ detected by Fourier transform infrared spectroscopy, which have tentatively been associated with FeIn acceptor states. ${ }^{42}$ However, our experimental data cannot definitely rule out the possibility that the deep donor level at $E_{\nu}+0.27 \mathrm{eV}$ might be the dominant FeIn-related recombination center.

After illuminating the Fe-contaminated In-doped $\mathrm{Si}$ wafer for $1 \mathrm{~min}$ at $0.7 \mathrm{~W} / \mathrm{cm}^{2}$, the lifetime in Fig. 5 shows a pronounced increase. The measured injection-dependent lifetime curve after light soaking (closed squares in Fig. 5) can be accurately fitted by assuming that all FeIn pairs have been dissociated during illumination and that isolated $\mathrm{Fe}_{i}$ alone determines the recombination lifetime. We conclude that FeIn pairs show a light-stimulated dissociation into $\mathrm{Fe}_{i}$ and $\mathrm{In}_{s}$ equivalent to that observed for $\mathrm{FeGa}$ and $\mathrm{FeB}$ pairs. Our results therefore indicate that light-stimulated dissociation is a common property of all iron-acceptor pairs. 


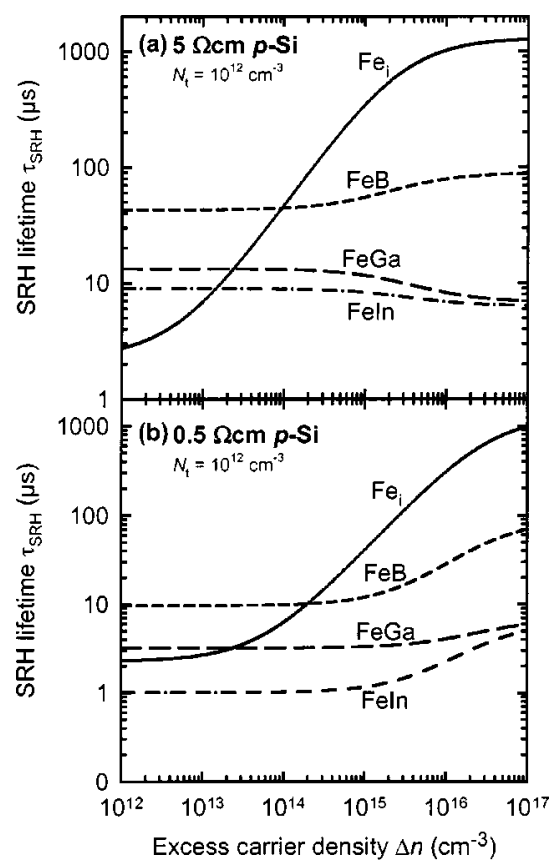

FIG. 6. Injection-dependent $\mathrm{SRH}$ lifetime curves for $\mathrm{Fe}_{i}, \mathrm{FeB}, \mathrm{FeGa}$, and FeIn in (a) $5-\Omega \mathrm{cm}$ and (b) $0.5-\Omega \mathrm{cm} p$-type $\mathrm{Si}$ calculated for $T=300 \mathrm{~K}$ using the defect parameters given in Table II. The density of centers has been taken as $N_{t}=10^{12} \mathrm{~cm}^{-3}$.

\section{Comparison of different iron-acceptor pairs}

In order to directly compare the recombination activity of different iron-acceptor pairs in $p$-type silicon, we have calculated the injection-dependent SRH lifetimes for a fixed defect concentration of $N_{t}=10^{12} \mathrm{~cm}^{-3}$ using Eq. (1). The defect parameters for $\mathrm{Fe}_{i}$ and $\mathrm{FeB}$ were taken from the literature, ${ }^{11,26}$ whereas the parameters for FeGa and FeIn have been determined in this study. All defect parameters used in the calculations are compiled in Table II. Figure 6 shows the calculated SRH lifetimes associated with each type of recombination center in high-resistivity $(5 \Omega \mathrm{cm})$ and low-resistivity $(0.5 \Omega \mathrm{cm}) p$-type silicon. As can clearly be seen from Fig. 6, independent of doping concentration and injection level, $\mathrm{FeGa}$ and FeIn pairs are much more effective recombination centers than $\mathrm{FeB}$ pairs. The comparison of $\mathrm{FeGa}$ and FeIn is more difficult because the uncertainty in the defect parameters determined for FeIn is relatively large due to the fact that we have only studied In-doped Si of one specific relatively high resistivity, showing similar recombination lifetimes as the corresponding Ga-doped material. Hence, FeGa and FeIn pairs show a comparable recombination activity in high-resistivity $p$-type silicon [see Fig. 6(a)]. In low-resistivity silicon, Fig. 6(b) indicates that FeIn shows a stronger recombination activity than $\mathrm{FeGa}$. However, it should be kept in mind that we have assumed here that all FeIn pairs are in the shallow-donor state at $E_{\nu}+0.15 \mathrm{eV} .^{27}$ The situation would be reversed if we assumed that the deepdonor state at $E_{\nu}+0.27 \mathrm{eV}$ determines the recombination lifetime of FeIn in $\mathrm{Si}^{27}$ However, this is relatively unlikely as the deep-donor state is known to be metastable, while the shallow-donor state corresponds to the stable FeIn configu- ration. Therefore, we conclude that it is likely that FeIn is the most effective recombination center of the three ironacceptor pairs studied.

After the complete dissociation of the iron-acceptor pairs, the recombination lifetime is determined only by interstitial iron (solid lines in Fig. 6), for which the defect parameters are relatively well known from the literature (see Table II). From dissociation experiments of $\mathrm{FeB}$ pairs in $p$-Si, it is known that there exists a particular excess carrier density $\Delta n_{\mathrm{COP}}$ at which the lifetime remains unchanged after dissociation. ${ }^{16,43}$ This crossover point of the injectiondependent lifetime curves associated with $\mathrm{FeB}$ and $\mathrm{Fe}_{i}$, respectively, (solid and short-dashed lines in Fig. 6) has recently been shown to increase linearly with doping concentration. ${ }^{26}$ It was also found that $\Delta n_{\mathrm{COP}}>10^{14} \mathrm{~cm}^{-3}$, and therefore iron-contaminated boron-doped silicon solar cells always degrade in efficiency after the dissociation of FeB pairs, because typical solar cells operate at excess carrier concentrations less than $10^{14} \mathrm{~cm}^{-3} \cdot{ }^{44}$ Interestingly, as under open-circuit conditions the excess carrier density can, depending on the type of solar cell and the degree of its contamination, be well above the crossover point, the opencircuit voltage of a solar cell may also increase in certain cases. ${ }^{44}$ Birkholz et al. ${ }^{26}$ have recently derived an analytical expression for the crossover point, which can also be applied to the dissociation of FeGa and FeIn pairs. This expression can be written in a simplified form for recombination centers with the energy level close to the valence-band edge and for doping concentrations $N_{A}>10^{14} \mathrm{~cm}^{-3}$ as

$$
\begin{aligned}
\Delta n_{\mathrm{COP}}= & \frac{\sigma_{n}^{-1}(\mathrm{Fe} A)-\sigma_{n}^{-1}\left(\mathrm{Fe}_{i}\right)}{\sigma_{p}^{-1}\left(\mathrm{Fe}_{i}\right)} N_{A}+\frac{\sigma_{p}\left(\mathrm{Fe}_{i}\right)}{\sigma_{n}(\mathrm{Fe} A)} N_{v} \\
& \times \exp \left(-\frac{E_{t}(\mathrm{Fe} A)-E_{v}}{k T}\right),
\end{aligned}
$$

where $A$ stands for the acceptor atoms $\mathrm{Ga}$ and In. In the derivation we have also used the fact that $\sigma_{p}\left(\mathrm{Fe}_{i}\right)$ is several orders of magnitude smaller than all the other capture cross sections involved. As within the measurement uncertainty the electron-capture cross sections for $\mathrm{Fe}_{i}$ and $\mathrm{FeGa}$ are equal [i.e., $\sigma_{n}(\mathrm{FeGa})=\sigma_{n}\left(\mathrm{Fe}_{i}\right)$ ], according to Eq. (4) the crossover point does not depend on the doping concentration. Using Eq. (4) and the defect parameters from Table II, we calculate $\Delta n_{\mathrm{COP}}=2.5 \times 10^{13} \mathrm{~cm}^{-3}$ for Ga-doped $p$-Si, in agreement with Fig. 6. This crossover point is well below the $\Delta n_{\mathrm{COP}}$ of the FeB dissociation. The typical operating point of one-sun silicon solar cells is very close to the $\mathrm{FeGa}$ crossover point. Hence, we expect no or a very small change in efficiency of Fe-contaminated Ga-doped $\mathrm{Si}$ solar cells after the dissociation of FeGa pairs. For FeIn pairs the situation is very different. Because of the large electron-capture cross section for FeIn (see Table II), which is one order of magnitude larger than the electron-capture cross section for $\mathrm{Fe}_{i}$ [i.e., $\left.\sigma_{n}(\mathrm{FeIn}) \gg \sigma_{n}\left(\mathrm{Fe}_{i}\right)\right]$, the first term in Eq. (4) becomes negative. Hence, $\Delta n_{\mathrm{COP}}$ decreases with increasing doping concentration and even becomes negative for doping concentrations $N_{A}>1.3 \times 10^{16} \mathrm{~cm}^{-3}$, which means that there would exist no crossover point in low-resistivity In-doped $p$-Si, as shown in Fig. 6(b). 


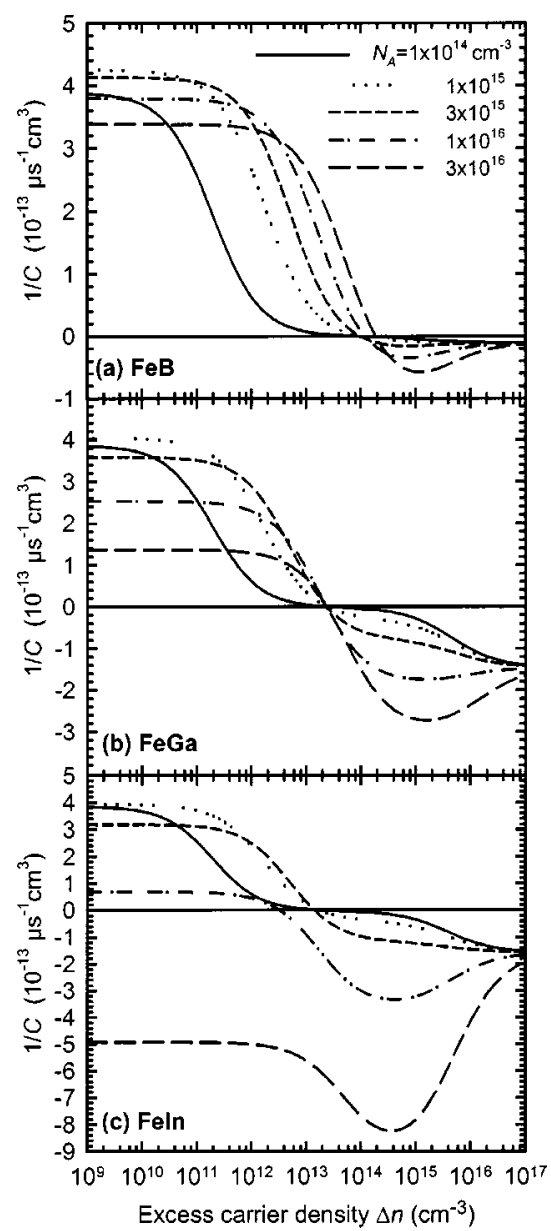

FIG. 7. Inverse calibration factor $1 / C$ as a function of excess carrier density $\Delta n$ and doping concentration $N_{A}$ calculated for $T=300 \mathrm{~K}$ for $p$-type silicon doped with (a) boron, (b) gallium, and (c) indium.

\section{Determination of iron concentration}

The most sensitive technique to measure the $\mathrm{Fe}_{i}$ concentration in B-doped $p$-type $\mathrm{Si}$ was proposed by Zoth and Bergholz. ${ }^{15}$ It is based on measuring the lifetimes (or diffusion lengths) before $\left(\tau_{0}\right)$ and after $\left(\tau_{1}\right)$ dissociation of FeB pairs. Assuming that all other recombination processes remain unchanged, the iron concentration can be determined by

$$
\left[\mathrm{Fe}_{i}\right]=C\left(\Delta n, N_{A}\right)\left(\frac{1}{\tau_{1}}-\frac{1}{\tau_{0}}\right),
$$

where the calibration factor $C$ is a function of the injection density $\Delta n$ and the doping concentration $N_{A}$ and can be easily calculated using Eq. (1). ${ }^{16}$ Using the defect parameters determined in this study for $\mathrm{FeGa}$ and FeIn, the same method can now also be applied to Ga- and In-doped $p$-type silicon. We have calculated the calibration factor $C\left(\Delta n, N_{A}\right)$ for all three iron-acceptor pairs considered in this study using the SRH equation and the defect parameters listed in Table II. The results are shown in Fig. 7. Negative values of $C$ are obtained for injection densities above the crossover point, meaning that the lifetime increases after dissociation. This case is most relevant for the determination of Fe concentrations by means of lifetime measurements as lifetime tech- niques usually operate under medium- to high-injection conditions. Alternatively, the diffusion length can be measured to determine the Fe content. Diffusion length measurement techniques, such as surface photovoltage, frequently operate at injection densities well below the crossover point. In this injection range, $C$ is usually positive and approaches a constant value for very low injection densities (see Fig. 7). As discussed above, the uncertainty is the largest for In-doped silicon shown in Fig. 7(c), whereas the curves shown in Figs. 7 (a) and 7(b) are based on very reliable data.

\section{CONCLUSIONS}

Recombination parameters for FeGa and FeIn pairs in silicon have been determined by fitting the SRH equation to injection-dependent carrier lifetime measurements performed on Ga- and In-doped $p$-type silicon wafers deliberately contaminated with iron. By using Ga-doped silicon wafers of different doping concentrations and low-injection lifetime data, it was possible to determine the energy level of the FeGa-related recombination center very accurately to be $E_{t}$ $=E_{\nu}+(0.20 \pm 0.01) \mathrm{eV}$ at room temperature, which is in good agreement with the literature data of the deep-donor state related to the stable trigonal configuration of $\mathrm{FeGa}$ in silicon. The relatively large electron-capture cross section $\sigma_{n}$ of 4 $\times 10^{-14} \mathrm{~cm}^{2}$ also determined from the low-injection lifetime data has been found to be the reason for the very strong recombination activity of $\mathrm{FeGa}$ pairs in silicon. A holecapture cross section $\sigma_{p}$ of $2 \times 10^{-14} \mathrm{~cm}^{2}$ has been obtained from the SRH fits to the complete injection-dependent lifetime curves. For the FeIn recombination center, the exact energy level could not be determined as In-doped silicon of only one resistivity was available in this study. However, the $\mathrm{SRH}$ fit to the measured injection-dependent lifetime data shows that the energy level is closer than $0.26 \mathrm{eV}$ to one of the band edges. In the case of FeIn, the shallow donor level at $E_{t}=E_{\nu}+0.15 \mathrm{eV}$ related to the orthorhomic configuration is known to be the stable state. Using this energy level, we obtain from the best fits to our injection-dependent lifetime data $\sigma_{n}=3.5 \times 10^{-13} \mathrm{~cm}^{2}$ and $\sigma_{p}=1.5 \times 10^{-14} \mathrm{~cm}^{2}$. Despite the relatively shallow energy level, its very large electroncapture cross section makes FeIn extremely recombination active. A direct comparison of the recombination activity of $\mathrm{FeB}, \mathrm{FeGa}$, and FeIn pairs in $p$-type silicon has shown that FeGa and FeIn pairs are much more effective recombination centers compared to FeB pairs.

Under illumination with white light, FeGa and FeIn were found to dissociate into interstitial iron and the corresponding substitutional acceptor. Storing the samples in the dark was found to lead to a full re-pairing of FeGa and FeIn pairs. We conclude that $\mathrm{FeGa}$ and FeIn pairs show a recombination-enhanced dissociation equivalent to that observed for FeB pairs, indicating that light-stimulated dissociation is a common property of all iron-acceptor pairs in silicon. Lifetime or diffusion length measurements performed before and after illumination can, hence, be used to determine the iron concentration in Ga- and In-doped silicon with a very high sensitivity. 


\section{ACKNOWLEDGMENTS}

J.S. thanks A. Cuevas and A. Blakers for their hospitality during his research stay at ANU and acknowledges the financial support of the Alexander von Humboldt Foundation. D.M. acknowledges the financial support of the Australian Research Council.

${ }^{1}$ H. Fischer and W. Pschunder, Proceedings of the Tenth IEEE Photovoltaic Specialists Conference, Palo Alto, California (IEEE, New York, 1973), p. 404.

${ }^{2}$ J. Schmidt, A. G. Aberle, and R. Hezel, Proceedings of the 26th IEEE Photovoltaic Specialists Conference, Anaheim, California (IEEE, New York, 1997), p. 13

${ }^{3}$ S. W. Glunz, S. Rein, W. Warta, J. Knobloch, and W. Wettling, Proceedings of the Second World Conference on Photovoltaic Solar Energy Conversion, Vienna, Austria (European Commission, Ispra, 1998), p. 1343.

${ }^{4}$ J. Schmidt and A. Cuevas, J. Appl. Phys. 86, 3175 (1999).

${ }^{5}$ J. Schmidt and K. Bothe, Phys. Rev. B 69, 024107 (2004).

${ }^{6}$ J. Schmidt, Solid State Phenom. 95-96, 187 (2004).

${ }^{7}$ T. Saitoh et al., Proceedings of the 16th European Photovoltaic Solar Energy Conference, Glasgow, United Kingdom (James \& James, London, 2000), p. 1206.

${ }^{8}$ S. W. Glunz, S. Rein, J. Y. Lee, and W. Warta, J. Appl. Phys. 90, 2397 (2001).

${ }^{9}$ A. Metz, T. Abe, and R. Hezel, Proceedings of the 16th European Photovoltaic Solar Energy Conference, Glasgow, United Kingdom (James \& James, London, 2000), p. 1189

${ }^{10}$ E. R. Weber, Appl. Phys. A 30, 1 (1983).

${ }^{11}$ A. A. Istratov, H. Hieslmair, and E. R. Weber, Appl. Phys. A 69, 13 (1999).

${ }^{12}$ T. F. Ciszek and T. H. Wang, J. Cryst. Growth 237-239, 1685 (2002).

${ }^{13}$ S. Beljakowa, D. Karg, G. Pensl, and J. Schmidt, Proceedings of the 19th European Photovoltaic Solar Energy Conference, Paris, France (WIPETA, Munich, 2004), p. 705.

${ }^{14}$ K. Graff and H. Pieper, J. Electrochem. Soc. 128, 669 (1981).

${ }^{15}$ G. Zoth and W. Bergholz, J. Appl. Phys. 67, 6764 (1990).

${ }^{16}$ D. H. Macdonald, L. J. Geerligs, and A. Azzizi, J. Appl. Phys. 95, 1021
(2004).

${ }^{17}$ G. W. Ludwig and H. H. Woodbury, Solid State Phys. 13, 223 (1962).

${ }^{18}$ H. Lemke, Phys. Status Solidi A 64, 215 (1981).

${ }^{19}$ L. V. C. Assali and J. R. Leite, Phys. Rev. B 36, 1296 (1987).

${ }^{20}$ S. Zhao, L. V. C. Assali, J. F. Justo, G. H. Gilmer, and L. C. Kimerling, J. Appl. Phys. 90, 2744 (2001).

${ }^{21}$ L. C. Kimerling, M. T. Asom, J. L. Benton, P. J. Drevinsky, and C. E. Caefer, Mater. Sci. Forum 38-41, 141 (1989).

${ }^{22}$ H. Lemke, Phys. Status Solidi A 76, 223 (1983).

${ }^{23}$ S. Zhao, L. V. C. Assali, and L. C. Kimerling, Mater. Sci. Forum 196201, 1333 (1995).

${ }^{24}$ Y. Hayamizu, T. Hamaguchi, S. Ushio, and T. Abe, J. Appl. Phys. 69, 3077 (1991)

${ }^{25}$ D. Macdonald, A. Cuevas, and J. Wong-Leung, J. Appl. Phys. 89, 7932 (2001).

${ }^{26}$ J. E. Birkholz, K. Bothe, D. Macdonald, and J. Schmidt, J. Appl. Phys. 97, 103708 (2005).

${ }^{27}$ A. Chantre and L. C. Kimerling, Mater. Sci. Forum 10-12, 387 (1986).

${ }^{28}$ T. Lauinger, J. Schmidt, A. G. Aberle, and R. Hezel, Appl. Phys. Lett. 68, 1232 (1996)

${ }^{29}$ R. A. Sinton and A. Cuevas, Appl. Phys. Lett. 69, 2510 (1996).

${ }^{30}$ H. Nagel, C. Berge, and A. G. Aberle, J. Appl. Phys. 86, 6212 (1999).

${ }^{31}$ D. Macdonald and A. Cuevas, Phys. Rev. B 67, 075203 (2003).

${ }^{32}$ W. Shockley and W. T. Read, Phys. Rev. 87, 835 (1952).

${ }^{33}$ R. N. Hall, Phys. Rev. 87, 387 (1952).

${ }^{34}$ J. S. Blakemore, Semiconductor Statistics (Dover, Mineola, 1987).

${ }^{35}$ W. M. Bullis and H. R. Huff, J. Electrochem. Soc. 143, 1399 (1996).

${ }^{36}$ M. A. Green, J. Appl. Phys. 67, 2944 (1990).

${ }^{37}$ L. C. Kimerling and J. L. Benton, Physica B \& C 116, 297 (1983).

${ }^{38}$ D. Macdonald and A. Cuevas, Appl. Phys. Lett. 74, 1710 (1999).

${ }^{39}$ J. Schmidt, K. Bothe, and R. Hezel, Appl. Phys. Lett. 80, 4395 (2002).

${ }^{40}$ K. Wünstel and P. Wagner, Appl. Phys. A 27, 207 (1982).

${ }^{41}$ K. Nakashima and M. Chijiiwa, Jpn. J. Appl. Phys., Part 1 25, 234 (1986)

${ }^{42}$ P. Tidlund, M. Kleverman, and H. G. Grimmeiss, Semicond. Sci. Technol 11, 748 (1996).

${ }^{43}$ A. A. Istratov, H. Hieslmair, and E. R. Weber, Appl. Phys. A 70, 489 (2000).

${ }^{44}$ J. Schmidt, Prog. Photovoltaics (in press). 